\title{
Design and experiment of a hydraulic lifting wind field test platform for crop protection UAS
}

\author{
Songchao Zhang ${ }^{1,2,3}$, Wei Gu ${ }^{3}$, Baijing Qiu ${ }^{1,2^{*}}$, Xinyu Xue ${ }^{3 *}$, Lixin Zhou ${ }^{3}$ \\ (1. Key Laboratory of Modern Agricultural Equipment and Technology, Ministry of Education, Jiangsu University, \\ Zhenjiang 212013, Jiangsu, China; \\ 2. Key Laboratory of Plant Protection Engineering, Ministry of Agriculture and Rural Affairs, Zhenjiang 212013, Jiangsu, China; \\ 3. Nanjing Institutes of Agricultural Mechanization, Ministry of Agriculture and Rural Affairs, Nanjing 210014, China)
}

\begin{abstract}
As a new type of crop protection machine, the crop protection unmanned aerial system (CPUAS) is developing rapidly in China. The wind field generated by the rotor has a great influence on the deposition and penetration of spraying droplets. The purpose of this study was to develop a reliable and stable test platform that could be used for wind field test of CPUAS, and to carry out the downwash experiments on the platform to obtain the downwash distribution law of a CPUAS Z-3N (100 kg level, Nanjing Research Institute on Simulation Technique, Nanjing, China). The tests showed that the performances of the developed platform could meet the expected design requirements. The platform operated stably and reliably during the downwash experiments of Z-3N, which indicated it could be applied for CPUASs of $100 \mathrm{~kg}$ level and below. The vibration characteristics of the platform with different heights $(2.0 \mathrm{~m}, 3.0 \mathrm{~m}, 5.0 \mathrm{~m}, 7.0 \mathrm{~m}, 10.0 \mathrm{~m})$ were obtained through modal analysis, which could effectively guide avoiding the resonance for stable and reliable operation during the experiments with the tested CPUAS Z-3N. A ring-radial method was designed combined with the platform for the downwash measurement. The experimental results showed that the downwash distribution of Z-3N was not symmetrical; the downwash wind speed decreased with the increase of the radial distance while the changing trend was not consistent as the height increased. Moreover, the area with high wind speed was mainly within $3.0 \mathrm{~m}$ of the radial distance, and the maximum value was $11.37 \mathrm{~m} / \mathrm{s}$. The study provided a new way for wind field test of CPUASs and would provide some references for better utilization of wind field during the CPUAS spraying.
\end{abstract}

Keywords: crop protection, unmanned aircraft system, wind field, test platform, vibration modal, aerial spraying DOI: $10.25165 /$ j.ijabe.20211404.6262

Citation: Zhang S C, Gu W, Qiu B J, Xue X Y, Zhou L X. Design and experiment of a hydraulic lifting wind field test platform for crop protection UAS. Int J Agric \& Biol Eng, 2021; 14(4): 166-174.

\section{Introduction}

The crop protection unmanned aerial system (CPUAS) has the advantages of superior mobility, wide adaptability, and high efficiency without the restrictions of the crop types or growth periods, especially suitable for the paddy fields and the mountainous $\operatorname{areas}^{[1-3]}$. In addition, compared with the traditional ground crop protection machines, the pesticide droplet deposition and penetration could be improved with the assistant of the downwash generated by the CPUAS rotors ${ }^{[4-8]}$, so as to achieve effective control of crop diseases and pests ${ }^{[9-11]}$. Therefore, the CPUAS has developed rapidly in China in recent years ${ }^{[12-15]}$, not only at the technical level but also the application area has been already the first around the world ${ }^{[16]}$. According to the 2018 statistics of the

Received date: 2020-11-05 Accepted date: 2021-05-08

Biographies: Songchao Zhang, MS, research interest: precise pesticide spraying, Email: zhangsongchao@qq.com; Wei Gu, MS, research interest: crop protection and machinery engineering, Email: guwei525@126.com; Lixin Zhou, MS, research interest: agricultural machinery engineering, Email: 525751791 @qq.com.

*Corresponding authors: Baijing Qiu, PhD, Professor, research interest: crop protection and machinery engineering. Key Laboratory of Modern Agricultural Equipment and Technology, Ministry of Education, Jiangsu University, Zhenjiang 212013, Jiangsu, China. Tel: +86-511-88797338, Email: qbj@ujs.edu.cn; Xinyu Xue, PhD, Professor, research interest: crop protection and machinery engineering. Nanjing Institute for Agricultural Mechanization, Ministry of Agriculture and Rural Affairs, Nanjing 210014, China. Tel: +86-25-84346243, Email: xuexynj@qq.com.
Chinese Ministry of Agriculture, the number of CPUAS was close to 30000 , and the application area was 17.3 million $\mathrm{hm}^{2[17]}$.

However, the droplet drift may be increased if the downwash was not utilized scientifically and rationally ${ }^{[18-20]}$, which would lead to pesticide waste and environmental pollution ${ }^{[16]}$. The coverage width, speed, and distribution of the downwash generated by the rotor are the main factors that affect the droplet deposition and the spray target percentage ${ }^{[21,22]}$, so the rotor wind field of the CPUAS and its scientific application has become the research focuses in the agricultural field. Scholars have conducted related researches on the distribution characteristics of the downwash of CPUAS in recent years. Yang et al. ${ }^{[6]}$ used the renormalization group (RNG) $k$ - $\omega$ turbulence model and dynamic grid technology to research the distribution characteristics of a six-rotor agricultural unmanned aerial vehicle (UAV) downwash in hover. The results showed that the characteristics of the airflow in the inlet and outlet regions of the adjacent rotor made the speed distribution of the downwash airflow significantly asymmetric. Jung et al. ${ }^{[23]}$ researched the mechanism of the interaction between the downwash and the tail fin of a dual rotor CPUAS and achieved an accurate computational fluid dynamics (CFD) simulation of the inlet and outlet regions of the downwash between the two rotors. Yoon et al. ${ }^{[24]}$ conducted a numerical simulation and carried out the experimental verification of interactional aerodynamics of multi-rotor flows on a quad-rotor CPUAS in hover, the results showed that the separation distances, as well as the wings, had significant effects on the vertical forces of quad-rotor systems in hover. Zheng et al. ${ }^{[25]}$ conducted a 
numerical simulation analysis of the downwash of a six-rotor CPUAS JF01-10 at different heights combined with sliding grid technology. The results showed that the more uniform the distribution of the downwash, the smaller the ground effect would be with the increase of the hover height, and the appropriate operational hovering height for the JF01-10 was considered to be $3.0 \mathrm{~m}$. Li et al. ${ }^{[26]}$ researched the mechanism of the interaction between the downwash of a UAV and the vortex generated in a rice canopy and constructed a follow-up model that included the movement parameters of the UAV body and the vortex movement of the rice canopy to improve the droplet target contact accuracy of the spraying operation of the UAV.

In terms of wind field measurement technology for the CPUAS, Wang et al. ${ }^{[27]}$ measured the speed of the downwash in the $x, y$, and $z$ directions, utilized a balanced distribution law of the spatial mass of the droplets and proved that the downwash had a significant impact on the behavior of the droplets and the downward airflow perpendicular to the ground direction could improve the deposition of the droplets. $\mathrm{Hu}$ et al. ${ }^{[28]}$ designed a three-dimensional wind field measurement system based on wireless sensor networks to measure the wind field distribution of agricultural UAV applied in hybrid rice pollination. The results proved the feasibility of the system and this study would provide a reference for the CPUAS wind field test. Wang et al. ${ }^{[2]}$ measured the airfield of an oil-powered single-rotor UAV working in a rice field by setting three direction sensors for wind speed measurement in a one-way linear array. The results showed that the fastest airflow occurred in the flying direction in parallel, followed by the speed of the airflow in the horizontal and perpendicular to the flying direction and the vertical direction, and the width of the downwash increased with a decrease in flight altitude. Based on machine vision, Pombeiro et al. ${ }^{[30]}$ researched the ripple characteristics caused by the downwash in the water surface of a multi-rotor UAV. Mylapore et al. ${ }^{[31]}$ directly observed the streamline of a rotor fuselage and the streamline distribution of the downwash on the ground. Guo et al. ${ }^{[32]}$ simulated and verified the spatial and temporal distributions of the downwash of a quad-rotor CPUAS in hover state, and the speed test experiment was designed to measure the wind field with an anemometer. Tang et al. ${ }^{[33]}$ developed an indoor measurement technique to investigate the downwash flow field of a 1:10 scaled unmanned agricultural helicopter, in which high-speed particle image velocimetry was employed to obtain the instantaneous and average speed field of the downwash.

In order to achieve a more scientific and reasonable application of the CPUAS, studies on the wind field especially the downwash are necessary and worthwhile for each type CPUAS. However, studies on the wind field of the CPUAS mainly focus on the spatial dimension, and scientific research achievements on the characteristics of rotor downwash in the temporal dimension are rarely reported. Furthermore, in terms of methods, researches are mainly based on computer simulations but rarely on the special test benches for measurement and verification. The computer simulation needs high accuracy models for different type CPUASs and the data obtained by the computer simulation are relatively ideal compared with the actual application. The field test would be affected by uncontrollable environmental factors such as the wind resulting in the inaccuracy of measurement. Therefore, a universal test platform meeting most of the CPUAS load requirements could be used to measure the wind field in a relatively stable environment. In this study, a new platform was developed based on the hydraulic lifting system, and an appropriate test

method was designed to test the Z-3N type CPUAS (Nanjing Research Institute on Simulation Technique, Nanjing, China) downwash distribution. This study will provide references for CPUAS wind field research and precise aerial spraying under the downwash.

\section{Materials and methods}

\subsection{Platform design}

\subsubsection{Design requirements}

The design purpose of the platform was to satisfy the wind field tests for most CPUASs applied currently in China. The platform should be able to carry the heaviest CPUAS in terms of load and could raise the CPUAS to different heights for simulating aerial spraying in different heights. Due to the vibrations caused by the CPUAS, the platform should avoid resonances inherently on certain frequencies at different heights. Furthermore, the platform could be driven by the ordinary civil power supply, easily moved and installed quickly for convenient tests.

2.1.2 Overall structure of the platform

The overall structure of the platform with a CPUAS is shown in Figure 1. The whole platform is composed of a power system, a lifting system, a fixed surface, and a stabilization system according to the functions. The power system drives the motor by introducing a $220 \mathrm{~V}$ alternating current (AC) power supply through the electrical control box. The lifting system includes an oil pump, telescopic cylinder, valve blocks, hydraulic oil, and oil tank, to raise the CPUAS to a required height. The fixed surface is an adjustable plane made of stainless steel connected with the lifting system through four bolts. The stabilization system consists of anchor bolts, screw fasteners, reinforced support plates, anti-rotation frames, and flexible fixed steel ropes, assisting to reduce vibrations and stabilize the test platform.

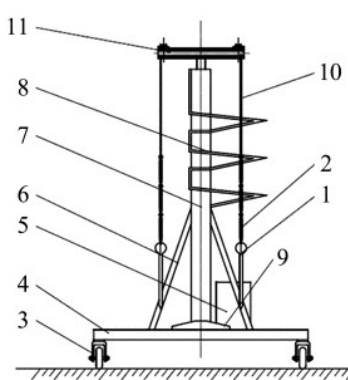

a. Main components of the platform

1. Anchor bolt 2. Screw fastener 3. Steering wheel 4. Base part 5. Electrical control box 6. Reinforced support plate 7. Telescopic cylinder 8. Anti-rotation frame 9. Hydraulic oil tank 10. Flexible fixed steel rope 11. CPUAS fixed surface 12. CPUAS

Figure 1 Overall structure of the platform with a CPUAS

\subsubsection{Working principle of the platform}

During operation, a remote-control handle is used to control the electric motor ( $220 \mathrm{~V} \mathrm{AC}$ ) providing the oil pump with a power supply for the platform rising and falling. The platform working principle is as follows. When the platform is rising, the hydraulic oil is drawn from the hydraulic oil tank under the action of the oil pump, through the oil filter and then through the electromagnetic directional valve to the lower piston cavity. The hydraulic oil in the upper piston cavity flows back to the oil tank. When falling, the hydraulic oil is drawn from the hydraulic oil tank under the action of the oil pump, through the oil filter and then through the electromagnetic directional valve to the upper piston cavity. The 
hydraulic oil in the lower piston cavity flows back to the oil tank. The valve blocks including the throttle valve, balance valve and overflow valve ensure the stability of platform movement in the process of hydraulic oil transport.

Figure 2 shows the hydraulic system working flowchart for the test platform rising and falling.

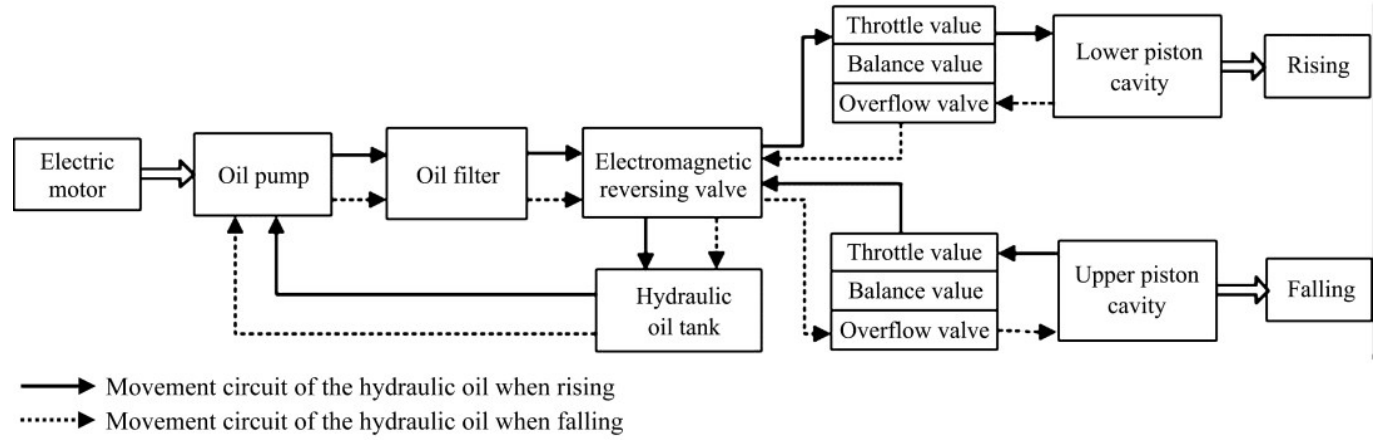

Figure 2 Working flowchart of hydraulic system for rising and falling of the test platform

\subsubsection{Main technical parameters}

According to the current applied situation in $\mathrm{China}^{[11-15,33,34]}$, the payloads of most CPUASs are less than $50 \mathrm{~kg}$, and the total weight of CPUAS is not more than $100 \mathrm{~kg}$. In practical aerial pesticide spraying, the CPUAS flight altitude is from $1.5 \mathrm{~m}$ to $7.0 \mathrm{~m}$ above the crops. The length of the CPUAS landing gear and the distance between them are generally $1.0 \mathrm{~m}$. Therefore, based on practical application and a certain margin reserved, the main platform parameter values have been determined (Table 1). The maximum rising height is $10.0 \mathrm{~m}$, the rated load is $200 \mathrm{~kg}$ $(220 \mathrm{~kg}$, Maximum), and the fixed surface is $800 \mathrm{~mm} \times 800 \mathrm{~mm}$ (1200 mm× $1200 \mathrm{~mm}$, Maximum).

Table 1 Main parameters of the platform

\begin{tabular}{lcc}
\hline \multicolumn{1}{c}{ Items } & Values & Remarks \\
\hline Size of base part $/ \mathrm{mm}$ & $1160 \times 1000 \times 2170$ & $/$ \\
Maximum rising height $/ \mathrm{mm}$ & 10000 & Relative to the base part \\
Rated load $/ \mathrm{kg}$ & 200 & $/$ \\
Rising time/s & 40 & 220V AC power source \\
Motor power $/ \mathrm{kW}$ & 1.1 & Adjustable \\
Size of the CPUAS fixed surface & $800 \times 800$ & $/$ \\
(mm $\times \mathrm{mm})$ & 700 & \\
Total weight/kg &
\end{tabular}

\subsection{Modal analysis of the platform}

\subsubsection{Vibration modal and test method}

The vibrations caused by the power output of the CPUAS exist during the wind field test. It is necessary to analyze the vibration characteristics of the platform to provide a reference for reasonable resonance avoidance in the test. The vibration modal analysis is a research method for structural dynamic characteristics, mainly including experimental modal analysis (EMA) and finite element analysis (FEA). EMA requires rich experiences for the tester, and the test period is relatively long, while in FEA when the structural parameters and the physical properties of the structural components are known, the analysis results could be drawn soon and meet the basic accuracy requirements generally ${ }^{[35-39]}$.

According to the FEA simulation theory, the component structures can be regarded as a vibrating structural system superimposed by multiple degrees of freedom, and the differential equation of its motion state is as follows ${ }^{[40]}$ :

$$
[M]\{: \cdots=\{f\}
$$

where, $M$ is the mass matrix; $K$ is the stiffness matrix; $C$ is the damping matrix; " is the acceleration; $\because$ is the velocity; $x$ is the displacement response column vector; $f$ is the added excitation force vector.
Equation (1) could be expressed by Equation (2) after Laplace transform.

$$
\left(s^{2}[M]+s[C]+[K]\right)\{X(s)\}=\{F(s)\}
$$

where, $X(s)$ is the displacement response; $F(s)$ is Laplace transform of the added excitation force; $s$ is the complex number variable in Laplace transform.

Let $s=j \omega$, then Equation (2) is expressed as follows:

$$
\left([K]-\omega^{2}[M]+j \omega[C]\right)\{X(j \omega)\}=\{F(j \omega)\}
$$

where, $j$ is the imaginary number; $j^{2}=-1 ; \omega$ is the frequency, $\mathrm{r} / \mathrm{s}$.

Equation (3) is coupled, which could be decoupled by introducing modal coordinate:

$$
\{X\}=[\phi]\{q\}
$$

where, $\phi$ is the modal matrix; $q$ is the modal coordinate. Equation (5) is obtained by taking Equation (4) into Equation (3) as follows:

$$
\left([K]-\omega^{2}[M]+j \omega[C]\right)[\phi]\{q\}=[F(j \omega)]
$$

The mass matrix $M$ and the stiffness matrix $K$ are diagonalized as follows based on the orthogonal characteristic relationship with the modal matrix $\phi$.

$$
[\phi]^{T}\left([K]-\omega^{2}[M]+j \omega[C]\right)[\phi]\{q\}=[\phi]^{T}\{F(j \omega)\}
$$

After orthogonal transformation, the $N$ degrees of freedom equation groups become independent $n$ degree of freedom equations, and the equation is as follows after decoupling:

$$
\left(k_{i}-\omega^{2} m_{i}+j \omega c_{i}\right) q_{i}=\sum_{l=1}^{N} \phi_{i l} F_{l} \quad(l=1,2, \ldots, n)
$$

where, $k_{i}$ is the $i$-th vector of the stiffness matrix $K ; m_{i}$ is the $i$-th vector of the mass matrix $M ; c_{i}$ is the $i$-th vector of the damping matrix.

It can be seen from Equation (7) that the modal superposition principle existing in the structure, that is, the responses of the multiple degrees of freedom system are the sum of responses of the single degree of freedom system with multiple modal coordinates after the orthogonal transformation.

\subsubsection{Simulation model setting}

The model was built with software ANSYS 14.5 on the basis of the platform's physical dimensions. The quadratic tetrahedron was chosen as the generic element type, the three dimensional ten-node solid structural element Solid 187 was chosen as the mechanical ANSYS parametric design language (APDL) unit which had a secondary displacement mode that could accurately simulate irregular models, and the element units were connected by common nodes. The base part was fixed to the ground restrictively. The material steel was chosen consistently with the actual platform. The main properties are listed in Table 2 . 
Table 2 Main properties of the material

\begin{tabular}{lc}
\hline \multicolumn{1}{c}{ Property } & Value \\
\hline Density $/ \mathrm{kg} \cdot \mathrm{m}^{-3}$ & 7850 \\
Young's modulus/Pa & $2 \times 10^{11}$ \\
Poisson's ratio/Pa & 0.30 \\
Bulk modulus $/ \mathrm{Pa}$ & $1.67 \times 10^{11}$ \\
Shear modulus $/ \mathrm{Pa}$ & $7.69 \times 10^{10}$ \\
\hline
\end{tabular}

\subsection{CPUAS down wash test}

\subsubsection{Test site and environmental condition}

The test platform was installed outdoors in the east laboratory of Nanjing Institute of Agricultural Mechanization (NIAM), Ministry of Agriculture and Rural Affairs $\left(118.886^{\circ} \mathrm{E}, 32.048^{\circ} \mathrm{N}\right)$, where was surrounded by a fence to ensure no intervention from non-tester and reduce the influence of external environmental factors (e.g., wind) during the test. The woods along the fence also provided a good windbreak for the test.

Considering the external wind was the most important factor affecting the downwash test, the environmental weather condition of the test site maintained good consistency during the test, and was recorded in Table 3.

Table 3 Test site weather conditions

\begin{tabular}{cccc}
\hline Weather factor & Items & Values & Remarks \\
\hline \multirow{2}{*}{ Wind } & & $2.58 \pm 0.12$ & Maximum \\
& Speed $/ \mathrm{m} \cdot \mathrm{s}^{-1}$ & $0.22 \pm 0.09$ & Minimum \\
& & $1.42 \pm 0.33$ & Average \\
\cline { 2 - 4 } & Direction $\left./{ }^{\circ}\right)$ & $0 \pm 12$ & Southeasterly \\
\hline Temperature $/{ }^{\circ} \mathrm{C}$ & $/$ & $32.0 \pm 0.5$ & Average \\
Relative humidity $/ \%$ & $/$ & $65 \pm 5$ & Average \\
\hline
\end{tabular}

\subsubsection{Tested CPUAS}

In order to verify the reliability and practicability of the platform, the Z-3N (Nanjing Research Institute on Simulation Technique, Nanjing, China) with a total weight of $100 \mathrm{~kg}$ was chosen as the tested CPUAS. Z-3N is a single-rotor oil-powered CPUAS. The main rotor diameter is $3.12 \mathrm{~m}$, the rated engine rotation speed is $6350 \mathrm{r} / \mathrm{min}$ (rotor rotation speed $828 \mathrm{r} / \mathrm{min}$ ), and the main excitation frequency is $105.8 \mathrm{~Hz}$. Figure 3 shows the $\mathrm{Z}-3 \mathrm{~N}$ and its installation on the platform.

The Kestrel 4500 anemometer (Nielsen-Kellerman company, US) was chosen as the measurement device with a maximum speed range of $40 \mathrm{~m} / \mathrm{s}$, resolution of $0.01 \mathrm{~m} / \mathrm{s}$, accuracy of $\pm 3 \%$. About 3000 data could be measured and stored in the internal memory. The data collected could be read out to the laptop by the Kestrel Interface for convenient analysis. The Kestrel 4500 was placed horizontally and set as an automatic storage mode.

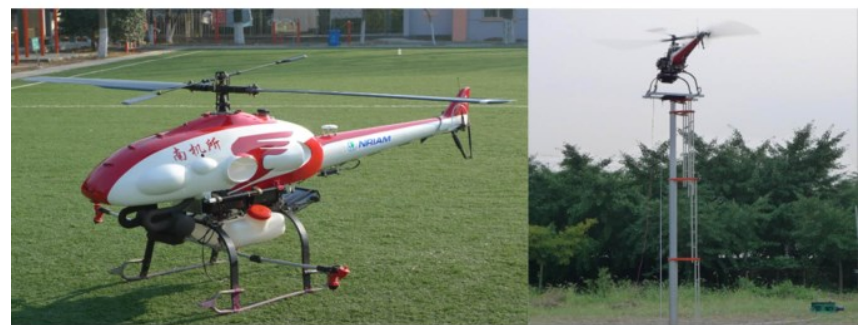

Figure 3 Tested CPUAS Z-3N and experimental installation

\subsubsection{Down wash test method}

Combined with the designed test platform, a ring-radial method was designed to measure the downwash. The coordinate system in the test was defined as shown in Figure $4 \mathrm{a}$. The intersection point of the CPUAS main rotor mast vertical downward direction with the ground was taken as the origin $O$ of the coordinate system, the vertically upward direction was set as the positive direction of the coordinate $Z$-axis, the fuselage forward direction was set as the positive direction of $Y$-axis and the direction of $90^{\circ}$ clockwise from the $Y$-axis was set as the positive direction of $X$-axis.

Eight directions $\left(O_{1}\right.$ to $\left.O_{8}\right)$ were set with $45^{\circ}$ interval from the positive $X$-axis counterclockwise and eight points $(a, b, c, d, e, f, g$, $h)$ with $0.5 \mathrm{~m}$ interval along each direction were marked from the origin $O$ on the ground (Figure 4). In actual CPUAS application, the heights of crops such as rice, wheat, soybean, corn, etc., are generally $0.5-2.0 \mathrm{~m}$, and the downwash during this height range is most concerned, so five measurement planes $\left(P_{1}, P_{2}, P_{3}, P_{4}, P_{5}\right)$ were set with an interval of $0.5 \mathrm{~m}$ vertically above corresponding ground marking points, and were the fixed positions of the anemometer Kestrel 4500 during the tests. In the experiments, the $\mathrm{Z}-3 \mathrm{~N}$ working on the rated condition was raised to $5.0 \mathrm{~m}, 6.0 \mathrm{~m}$ and $7.0 \mathrm{~m}$ above the ground according to the practical application. The downwash of each measurement point was recorded for $3 \mathrm{~min}$ after working stably, and the average value was taken as the effective wind speed.

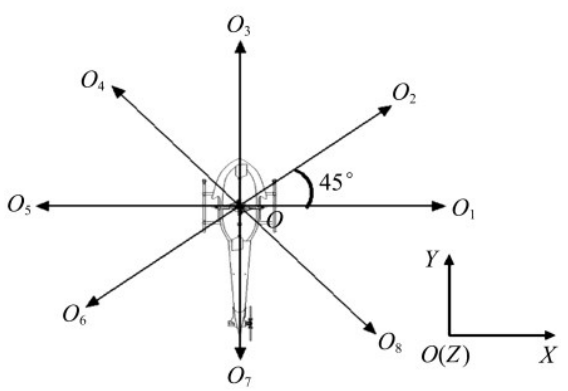

a. Top view

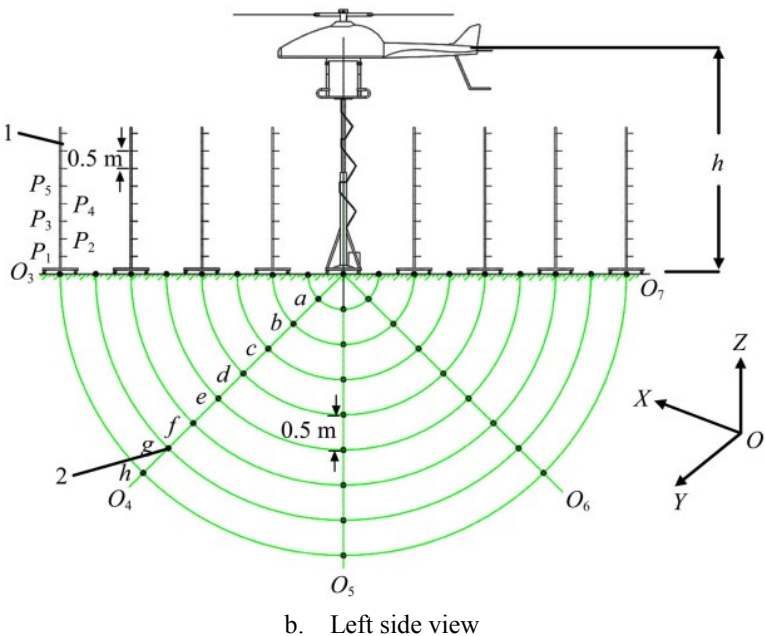

Figure 4 Test coordinate system and sampling point arrangements

\section{Results and analysis}

\subsection{Vibration modal results}

\subsubsection{Frequencies of different modes}

The FEA method was used to perform the platform modal analysis to the platform at heights of $2.0 \mathrm{~m}, 3.0 \mathrm{~m}, 5.0 \mathrm{~m}, 7.0 \mathrm{~m}$, and $10.0 \mathrm{~m}$, respectively. Since low-mode vibration energy accounts for a large proportion of vibration energy, and high-mode vibration energy accounts for a small proportion, ten order vibration modes were analyzed.

Taking the modal analysis of the platform at the height of $5.0 \mathrm{~m}$ as an example on account of paper space limitation, the total quantity of nodes was 390850 and the number of finite elements was 21658 in the simulation. Figure 5 shows the simulation 
vibration modal shapes of the platform when the platform was at a height of $5.0 \mathrm{~m}$ from ANSYS. It is consistent with the expectation that the inherent vibration frequency $(0.53 \mathrm{~Hz}, 4.40 \mathrm{~Hz}$, $4.40 \mathrm{~Hz}, 20.6 \mathrm{~Hz}, 38.7 \mathrm{~Hz}, 39.3 \mathrm{~Hz}, 40.1 \mathrm{~Hz}, 43.7 \mathrm{~Hz}, 46.9 \mathrm{~Hz}$ and $67.5 \mathrm{~Hz}$ ) increases as the modal order increases. The resonances will occur if the external excitation frequencies are close to the inherent vibration frequencies and will cause system instability and damage to the structure. Based on the analysis results of each order mode at the $5.0 \mathrm{~m}$ height, the inherent vibration frequencies are far from the external excitation frequency $(105.8 \mathrm{~Hz})$, even if that of the tenth order mode also differs by $38.3 \mathrm{~Hz}$. Thus, carrying out the wind field test at this altitude is safe.

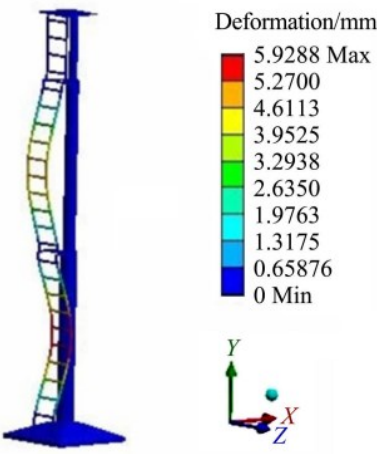

a. 1 st mode

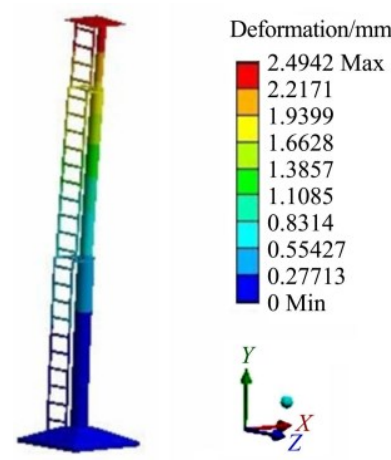

c. 3 rd mode

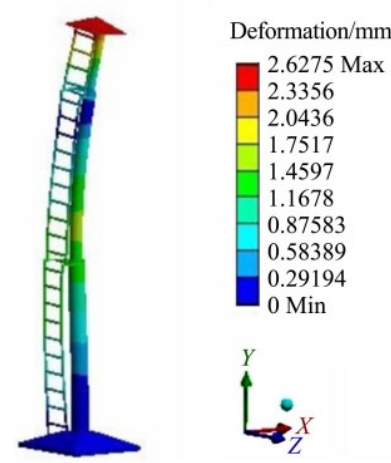

e. 5 th mode

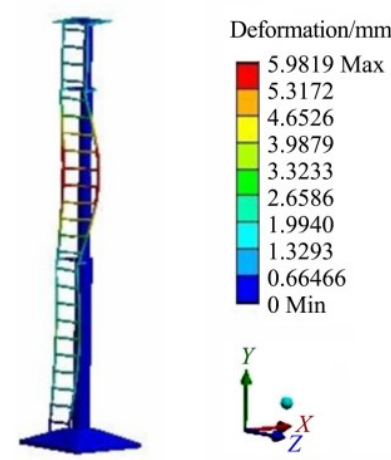

g. 7 th mode

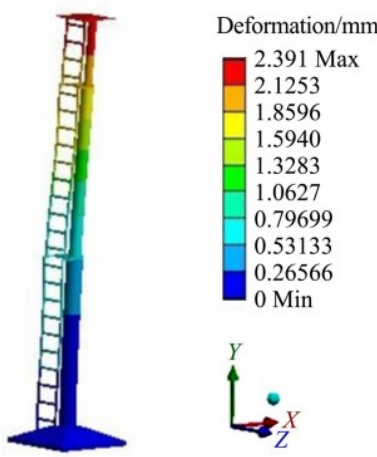

b. 2nd mode

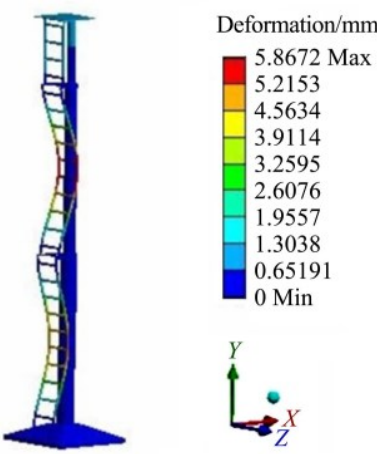

d. 4th mode

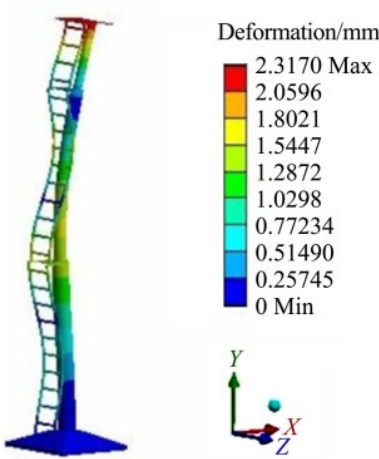

f. 6 th mode

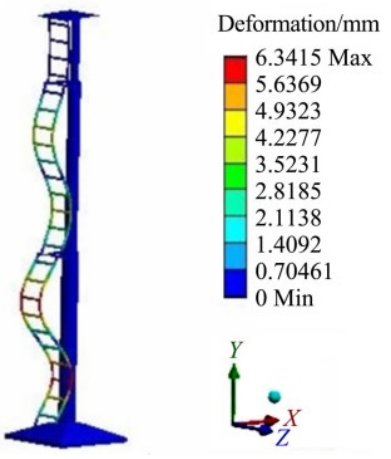

h. 8th mode

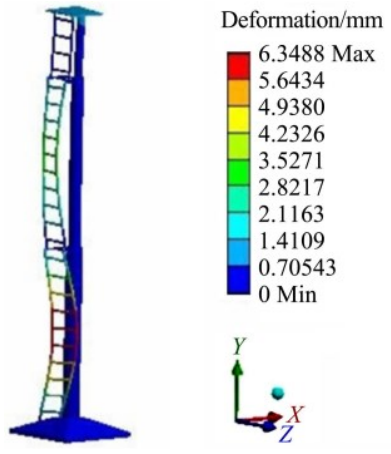

i. 9 th mode

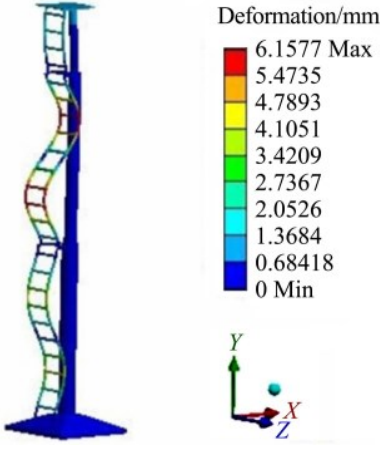

j. 10 th mode
Note: The 1st to 10th means the first-order mode to tenth order mode. The platform height of $5.0 \mathrm{~m}$

Figure 5 Ten order vibration modal shapes of the platform

The inherent vibration frequencies of the ten order modes results are shown in Table 4 . It can be seen from Table 4 that the first-order mode vibration frequencies of $2.0 \mathrm{~m}, 3.0 \mathrm{~m}, 5.0 \mathrm{~m}$, $7.0 \mathrm{~m}$ and $10.0 \mathrm{~m}$ are much lower than the excitation frequency, and the mode vibration frequencies below the seventh order mode are all lower than the excitation frequency. Therefore, it can be concluded that the resonances with the external excitation frequency are effectively avoided.

Table 4 FEA mode analysis results of the test platform

\begin{tabular}{|c|c|c|c|c|c|c|c|c|c|c|}
\hline \multirow{2}{*}{$\begin{array}{l}\text { Height } \\
/ \mathrm{m}\end{array}$} & \multicolumn{10}{|c|}{ Frequency/Hz } \\
\hline & $1 \mathrm{st}$ & 2nd & $3 \mathrm{rd}$ & 4th & 5 th & 6 th & 7 th & 8 th & 9th & 10th \\
\hline 2.0 & 1.32 & 20.60 & 36.30 & 37.60 & 40.10 & 71.90 & 78.50 & 101.90 & 114.60 & 124.50 \\
\hline 3.0 & 3.35 & 12.10 & 12.20 & 20.60 & 40.10 & 42.70 & 46.80 & 81.40 & 86.70 & 87.30 \\
\hline 5.0 & 0.53 & 4.40 & 4.40 & 20.60 & 38.70 & 39.30 & 40.10 & 43.70 & 47.50 & 67.50 \\
\hline 7.0 & 0.40 & 2.90 & 3.00 & 20.60 & 21.30 & 21.40 & 40.10 & 42.70 & 46.90 & 61.70 \\
\hline 10.0 & 0.30 & 2.00 & 2.10 & 9.96 & 9.98 & 20.60 & 27.80 & 27.90 & 40.60 & 42.80 \\
\hline
\end{tabular}

3.1.2 Strength and the mode shape

The four bolts are the key components for the fixed surface connected to the platform. The strength limit of the bolts should be greater than the impact strength borne during the tests. The modal analysis results show that the maximum impact strength on the bolt is about $34 \mathrm{MPa}$, which is far less than its strength limit of $450 \mathrm{MPa}$. The strength can fully satisfy the test safety requirements.

In terms of the mode shape, the most concerned ones are on or near the external excitation frequency of $105.8 \mathrm{~Hz}$. In the ten order vibration modes, only the frequencies of the eighth and ninth order modes at the height of $2.0 \mathrm{~m}$ are close to the external excitation frequency shown in Table 4, while the modal analysis results show that the mode shapes are mainly vertical on these two order modes, which means few influences on the stability of the platform.

\subsection{Down wash of the wind field}

\subsubsection{Down wash standard deviation analysis}

The standard deviation of the measured downwash wind speeds at $5.0 \mathrm{~m}, 6.0 \mathrm{~m}$ and $7.0 \mathrm{~m}$ heights was calculated according to the equation below.

$$
\sigma=\sqrt{\frac{1}{N} \sum_{i=1}^{N}\left(x_{i}-u\right)^{2}}
$$

where, $\sigma$ is the standard deviation, $\mathrm{m} / \mathrm{s} ; N$ is the number of the samples; $x_{i}$ is the wind speed value of a sample, $\mathrm{m} / \mathrm{s} ; u$ is the average wind speed value of samples, $\mathrm{m} / \mathrm{s}$. The data dispersion was evaluated by the downwash wind speed standard deviations calculated on the eight points $(a, b, c, d, e, f, g, h)$ of each direction $\left(O_{1}\right.$ to $\left.O_{8}\right)$ and each measurement plane $\left(P_{1}\right.$ to $\left.P_{5}\right)$ under three 
heights $(5.0 \mathrm{~m}, 6.0 \mathrm{~m}, 7.0 \mathrm{~m})$. The downwash speed standard deviations of different sampling positions on five planes when the CPUAS on the platform with different heights are shown in Figure 6.

As Figure 6 shown, most of the standard deviations are less than $2.0 \mathrm{~m} / \mathrm{s}$, indicating the reliability of the obtained data. Further analysis can be obtained that the overall standard deviations of the three heights were all less than $1.0 \mathrm{~m} / \mathrm{s}$. The dispersion of data obtained when CPUAS was at the height of $5.0 \mathrm{~m}$ shown in Appendix was smallest, which implied a more accurate representation for the downwash speed distribution. Therefore, the data obtained when CPUAS was at the height of $5.0 \mathrm{~m}$ were analyzed in the following sections.

\subsubsection{Down wash speeds variation with vertical distance}

Figure 7 shows the downwash speeds variation with the vertical distance at different sampling points $(a, b, c, d, e, f, g, h)$ when the CPUAS was raised to $5.0 \mathrm{~m}$.

It can be drawn that the downwash is not symmetrically combined with the position setting of the sampling points, which is consistent with the conclusion of Yang et al. ${ }^{[6]}$. The wind speeds do not vary consistently, some wind speed curves in eight directions show a trend of the first decline and then rise with the increase of height. The extreme wind speed of each point appears at the height of $2.0 \mathrm{~m}\left(O_{5^{-}}-a, O_{5}-b, O_{5^{-}} c, O_{5^{-}} d, O_{6}-e, O_{5}-f, O_{1}-g\right.$, $\left.O_{1}-h\right)$. The curves appearing concave at about $1.0 \mathrm{~m}$ height indicate the downwash occurred at a local minimal interval at about $1.0 \mathrm{~m}$ height. At most sampling points, the wind speeds in $O_{1}, O_{5}$ and $O_{6}$ directions are generally higher while those in $O_{4}$ direction are generally lower. The wind speeds at the sampling points with the same radial distance and vertical distance are different, and the maximum difference value exceeds $10.00 \mathrm{~m} / \mathrm{s}\left(2.0 \mathrm{~m}\right.$ height, $O_{5^{-}} d$ and $O_{4}-d$ ).

\subsubsection{Down wash speeds variation with radial distance}

Figure 8 shows downwash speed variations in the direction $O_{1}$ to $O_{8}$ when the CPUAS was raised to $5.0 \mathrm{~m}$ at the measuring heights of $0.5 \mathrm{~m}, 1.0 \mathrm{~m}, 1.5 \mathrm{~m}, 2.0 \mathrm{~m}$ and $2.5 \mathrm{~m}$, respectively.

As Figure 8 shown, the wind speeds of the downwash generally show a downward trend as the radial distance increases at the same height, which is especially obvious after the radial distance exceeds $2.0 \mathrm{~m}$. The wind speed extreme values in each direction are different, and most of the maximum wind speeds appear at the radial distance of $0.5-2.0 \mathrm{~m}$. At the height of $0.5 \mathrm{~m}$, due to the influence of the ground, the volatility of the wind speed is greater than that at other heights. The maximum value of the downwash speed is $11.37 \mathrm{~m} / \mathrm{s}\left(O_{5}-d, 2.0 \mathrm{~m}\right.$ height $)$. The higher downwash speeds distribute at the radial distance of 0.5-3.0 m, and most of the downwash speed values are greater than $0.50 \mathrm{~m} / \mathrm{s}$ within the radial distance of $3.0 \mathrm{~m}$.
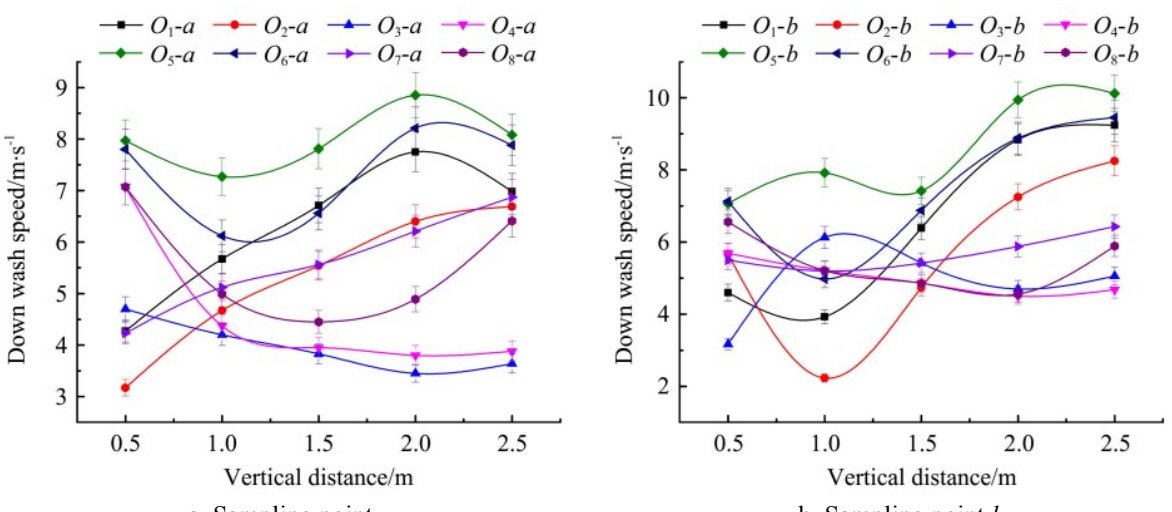

b. Sampling point $b$

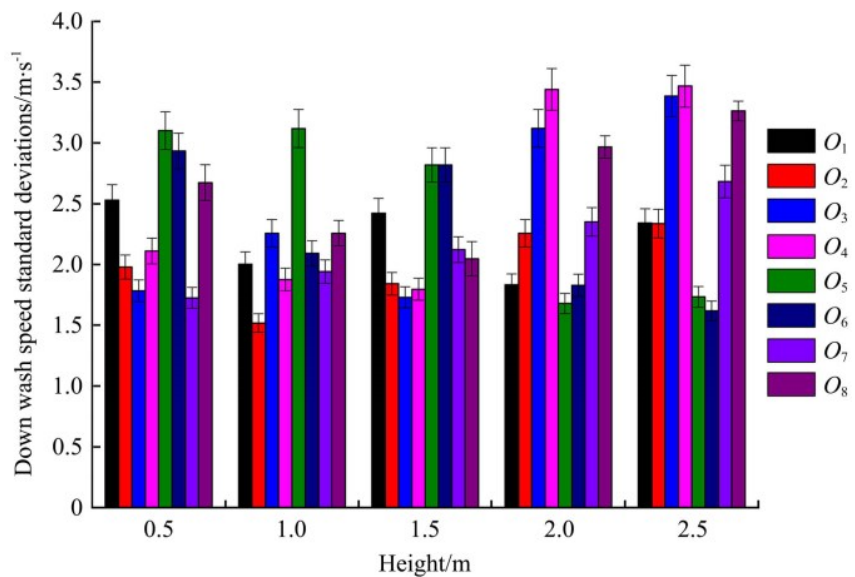

a. $h=5.0 \mathrm{~m}$

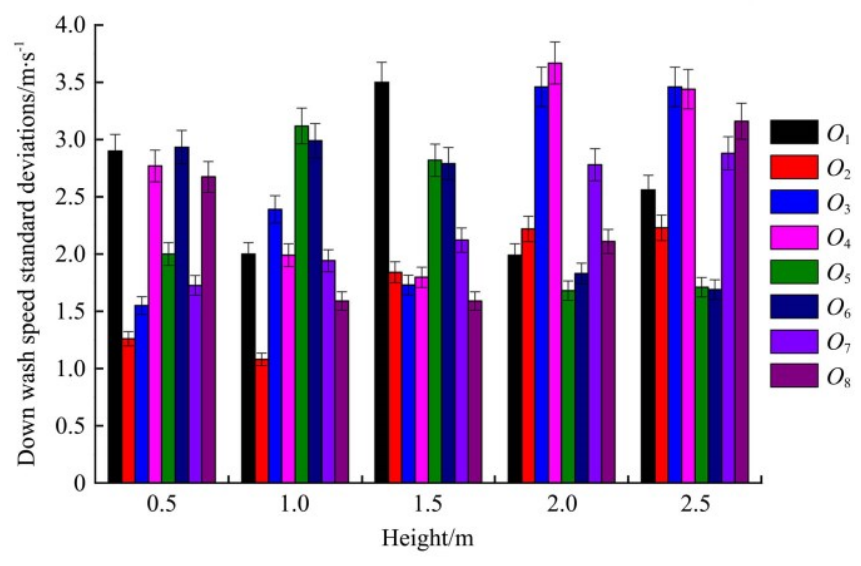

b. $h=6.0 \mathrm{~m}$

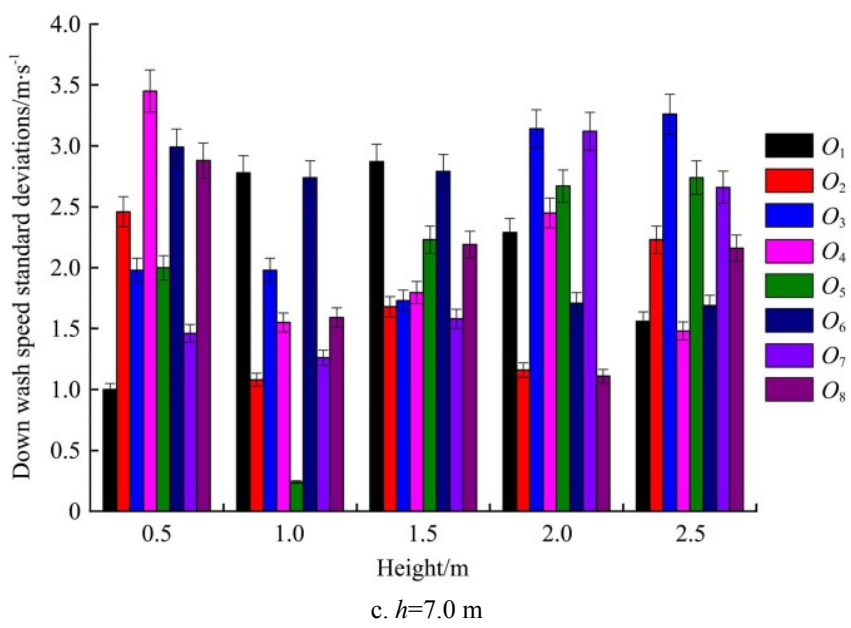

Note: $h$ represents the height of the CPUAS on the platform during the tests.

Figure 6 Down wash wind speed standard deviations of different sampling positions on five planes

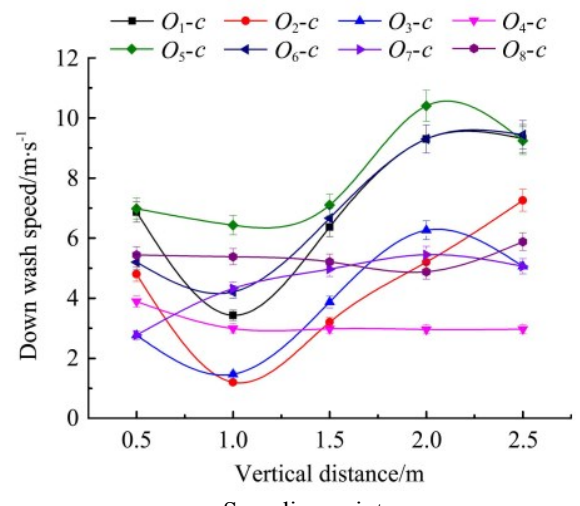

c. Sampling point $c$ 


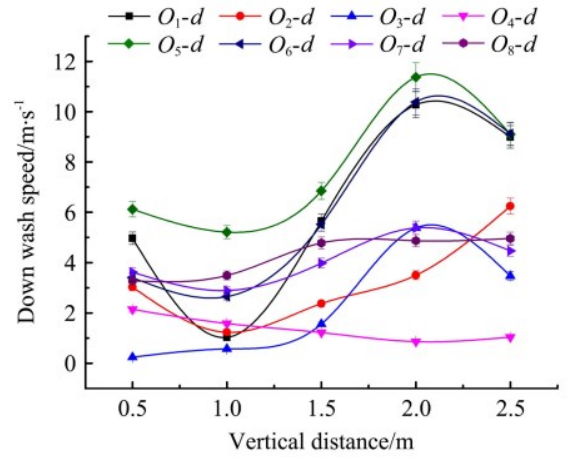

d. Sampling point $d$

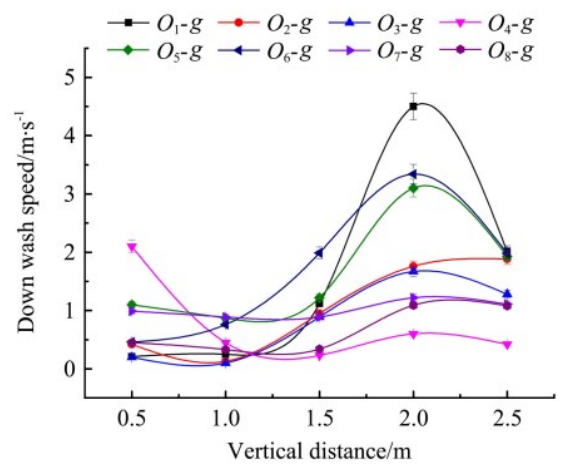

g. Sampling point $g$

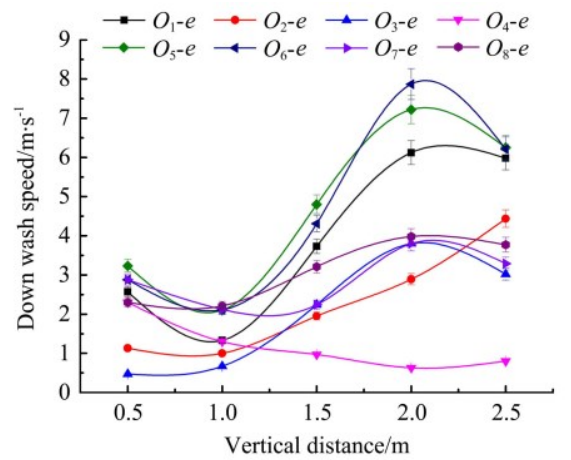

e. Sampling point $e$

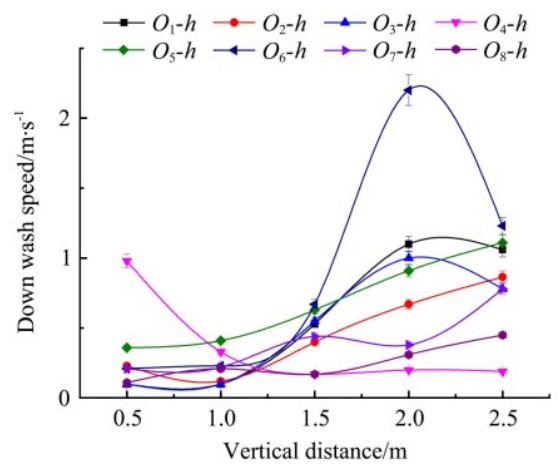

h. Sampling point $h$

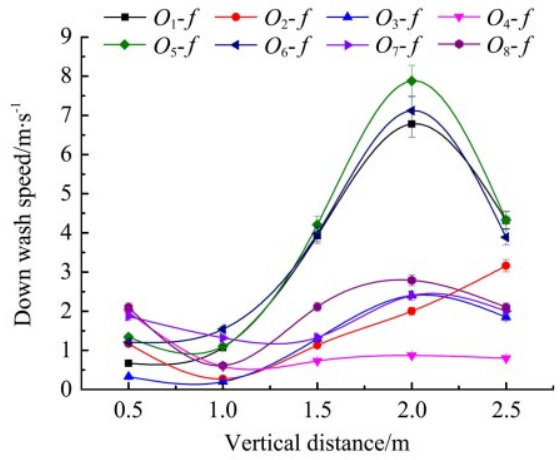

f. Sampling point $f$

Figure 7 Down wash speed variations with the vertical distance at different sampling points

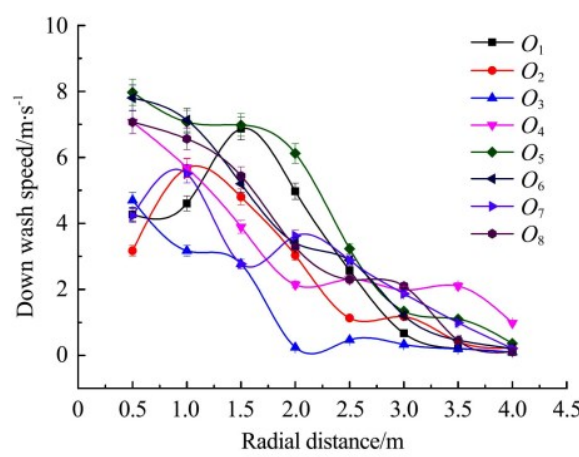

a. $0.5 \mathrm{~m}$ height

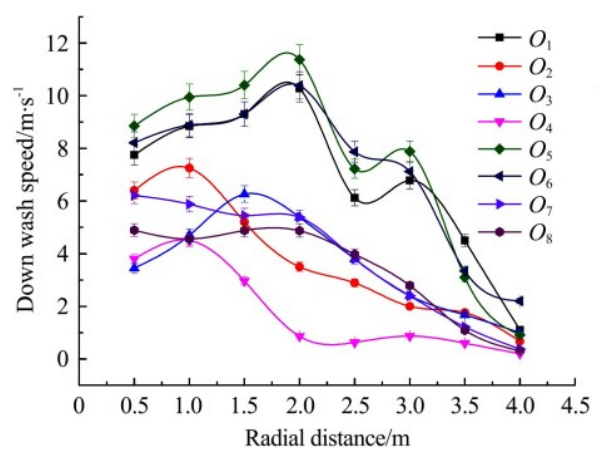

d. $2.0 \mathrm{~m}$ height

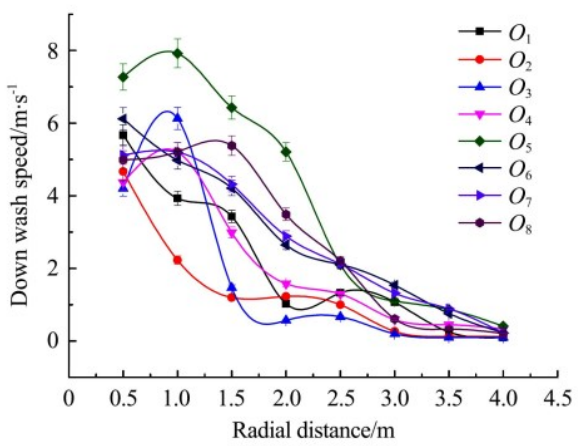

b. $1.0 \mathrm{~m}$ height

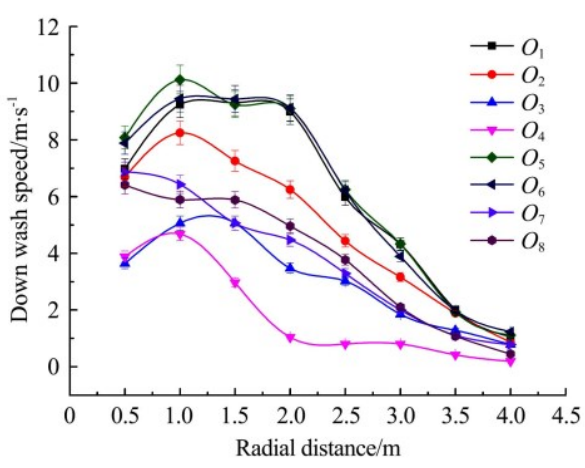

e. $2.5 \mathrm{~m}$ height

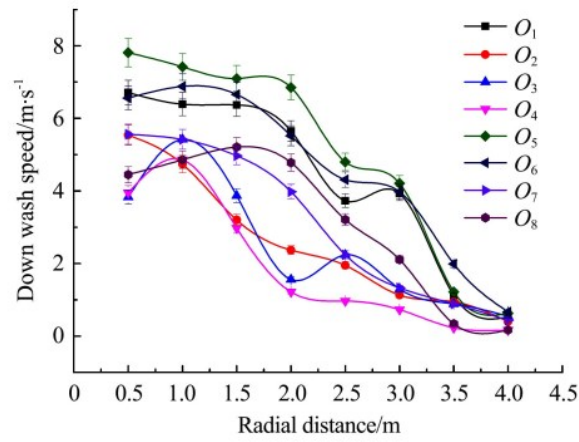

c. $1.5 \mathrm{~m}$ height

Figure 8 Down wash speed variations with the radial distance at different heights

\section{Conclusions}

In this study, a hydraulic lifting wind field test platform for CPUAS was developed and vibration modes of the test platform were analyzed. The downwash of a $100 \mathrm{~kg}$ CPUAS was tested and analyzed. The conclusions are shown as follows:

1) The hydraulic lifting wind field test platform was operating safely and smoothly, which proved that the stability and reliability of the platform could satisfy the design requirements.
2) The platform could satisfy the wind field test requirements of Z-3N CPUAS with nearly the maximum weight of $100 \mathrm{~kg}$, which indicated that it could be applied to other type CPUASs with relatively small loads in use currently.

3) The FEA vibration modal analysis could be used for the vibration characteristics of the platform working on different heights $(2.0 \mathrm{~m}, 3.0 \mathrm{~m}, 5.0 \mathrm{~m}, 7.0 \mathrm{~m}, 10.0 \mathrm{~m})$, and the experiments also verified that the platform could avoid resonances during the $\mathrm{Z}-3 \mathrm{~N}$ downwash testing at $5.0 \mathrm{~m}$ and $7.0 \mathrm{~m}$. 
4) The downwash distribution was not symmetrical. The wind speeds were relatively higher in direction $O_{1}, O_{5}$ and $O_{6}$, while that were relatively lower in direction $O_{4}$ generally. At the same sampling point, the wind speeds do not vary consistently, some wind speed curves in eight directions show a trend of the first decline and then rise with the increase of height, and the maximum appeared at the height of about $2.0 \mathrm{~m}$, which should be considered when high droplet penetration was required in aerial spraying application.

5) At the same height, the downwash wind speed shows a decreasing trend as the radial distance increases. Taking $0.5 \mathrm{~m} / \mathrm{s}$ as the boundary speed, the area with high wind speed was approximately within the radial distance of $3.0 \mathrm{~m}$, which was about two times the rotor radius.

In this study, although the experiment demonstrated the feasibility of the platform, for safety concerns, modal analysis of different heights should be performed for other CPUAS wind field testing according to the external excitation frequency (engine or motor). In this study, the wind field test was focusing on the downwash which had the greatest impact on the droplet deposition. As the wind field generated by the CPUAS rotors is vectorial, an effective measure, such as spatial triaxial test will be needed to improve spatial vector description accuracy in the future. The results of this study could be used as a reference.

\section{Acknowledgements}

This research was financially supported by the National Key Research and Development Program of China (Grant No. 2017YFD0701000), the National Natural Science Foundation of China (Grant No. 31701327), the China Agriculture Research System of MOF and MARA (Grant No. CARS-12), the Agricultural Science and Technology Innovation Project of the Chinese Academy of Agricultural Sciences, Crop Protection Machinery Team (Grant No. CAAS-ASTIP-CPMT), the Jiangsu Science and Technology Development Plan (Grant No. BE2019305), the Science and Technology Development Plan of Suzhou, Jiangsu Province (Grant No. SNG2020042).

\section{[References]}

[1] Xue X Y, Qin W C, Sun Z, Zhang S C, Zhou L X, Wu P. Effects of N-3 UAV spraying methods on the efficiency of insecticides against planthoppers and Cnaphalocrocis medinalis. Acta Phytophylacica Sinica, 2013; 40(3): 273-278. (in Chinese)

[2] Mozhdeh S. Jérôme T, Patrick M. Recent applications of unmanned aerial imagery in natural resource management. Giscience \& Remote Sensing, 2014; 51(4): 339-365.

[3] Qin W C, Qiu B J, Xue X Y, Chen C, Xu Z F, Zhou Q Q. Droplet deposition and control effect of insecticides sprayed with an unmanned aerial vehicle against plant hoppers. Crop Protection, 2016; 85: 79-88.

[4] Koo Y M, Bae Y H, Seok T S, Shin S K, Park H J. Tail rotor design and thrust test for a roll-balanced agricultural unmanned helicopter. Journal of Biosystems Engineering, 2010; 35(5): 302-309.

[5] Li J Y, Guo S, Yao W X, Zhan Y L, Li Y F. Distribution characteristics of droplet size in rice field and wind tunnel simulation test under airflow operation. Transactions of the CSAM, 2019; 50(8): 148-156. (in Chinese)

[6] Yang F B, Xue X Y, Zhang L, Sun Z. Numerical simulation and experimental verification on downwash air flow of six-rotor agricultural unmanned aerial vehicle in hover. Int J Agric \& Biol Eng, 2017; 10(4): $41-53$.

[7] Liao J, Zang Y, Luo X W, Zhou Z Y, Lan Y B, Zang Y, et al. Optimization of variables for maximizing efficacy and efficiency in aerial spray application to cotton using unmanned aerial systems. Int J Agric \& Biol Eng, 2019; 12(2): 10-17.

[8] Wang G B, Lan Y B, Qi H X, Chen P C, Hewitt A J, Han Y X. Field evaluation of an unmanned aerial vehicle (UAV) sprayer, effect of spray volume on deposition and the control of pests and disease in wheat. Pest Management Science, 2019; 75(6): 1546-1555.
[9] Chen S D, Lan Y B, Li J Y, Zhou Z Y, Liu A M, Mao Y. Effect of wind field below unmanned helicopter on droplet deposition distribution of aerial spraying. Int J Agric \& Biol Eng, 2017; 10(3): 67-77.

[10] Chen P C, Lan Y B, Huang X Y, Qi H X, Wang G B, Wang J, et al. Droplet deposition and control of plant hoppers of different nozzles in two-stage rice with a quadrotor unmanned aerial vehicle. Agronomy, 2020; 10(2): 303. doi: 10.3390/agronomy10020303.

[11] Lou Z X, Xin F, Han X Q, Lan Y B, Duan T Z, Fu W. Effect of unmanned aerial vehicle flight height on droplet distribution, drift and control of cotton aphids and spider mites. Agronomy, 2018; 8(9): 187. doi: 10.3390/agronomy8090187.

[12] Xue X Y, Gu W, Xu Y, Sun Z, Lan Y B. Review on current state of agricultural UAS regulations and standards. Transactions of the CSAM, 2020; 51(10): 1-10. (in Chinese)

[13] Zhou Z Y, Ming R, Zang Y, He X G, Luo X W, Lan Y B. Development status and countermeasures of agricultural aviation in China. Transactions of the CSAE, 2017; 33(20): 1-13. (in Chinese)

[14] Yang S L, Yang X B, Mo J Y. The application of unmanned aircraft systems to plant protection in China. Precision Agriculture, 2018; 19: 278-292.

[15] He X K, Bonds J, Herbst A, Langenakens J. Recent development of unmanned aerial vehicle for plant protection in East Asia. Int J Agric \& Biol Eng, 2017; 10(3): 18-30.

[16] Zhang S C, Qiu B J, Xue X Y, Sun T, Peng B. Parameters optimization of crop protection UAS based on the first industry standard of China. Int J Agric \& Biol Eng, 2020; 13(3): 29-35.

[17] Wang G B, Han Y X, Li X, Andaloro J, Lan Y B. Field evaluation of spray drift and environmental impact using an agricultural unmanned aerial vehicle (UAV) sprayer. Science of The Total Environment, 2020; 737: 139793. doi: 10.1016/j.scitotenv.2020.139793.

[18] Zhang S C, Xue X Y, Qin W C, Sun Z, Ding S M, Zhou L X. Simulation and experimental verification of aerial spraying drift on $\mathrm{N}-3$ unmanned spraying helicopter. Transactions of the CSAE, 2015; 31(3), 87-93. (in Chinese)

[19] Yang F B, Xue X Y, Cai C, Zhou Q Q. Effect of down wash airflow in hover on droplet motion law for multi-rotor unmanned plant protection machine. Transactions of the CSAE, 2018; 34(2): 64-73. (in Chinese)

[20] Xue X Y, Tu K, Qin W C, Lan Y B, Zhang H H. Drift and deposition of ultra-low altitude and low volume application in paddy field. Int J Agric \& Biol Eng, 2014; 7(4): 23-28.

[21] Tang Q, Zhang R R, Chen L P, Xu M, Zhang B. Droplets movement and deposition of an eight-rotor agricultural UAV in downwash flow field. Int J Agric \& Biol Eng, 2017; 10(3): 47-56.

[22] Lyu M Q, Xiao S P, Tang Y, He Y. Influence of UAV flight speed on droplet deposition characteristics with the application of infrared thermal imaging. Int J Agric \& Biol Eng, 2019; 12(3): 10-17.

[23] Jung Y S, You J Y, Kwon O J. Numerical investigation of prop-rotor and tail-wing aerodynamic interference for a tilt-rotor UAV configuration. Journal of Mechanical Science and Technology, 2014; 28(7): 2609-2617.

[24] Yoon S, Lee H C, Pulliam T H. Computational analysis of multi-rotor flows. In: American Institute of Aeronautics and Astronautics, 54th Aerospace Sciences Meeting, San Diego, USA, 2016; pp.812-823.

[25] Zheng Y J, Yang S H, Liu X X, Wang J, Norton T, Chen J, et al. The computational fluid dynamic modeling of downwash flow field for a six-rotor UAV. Frontiers of Agricultural Science and Engineering, 2018; 5(2): 159-167.

[26] Li J Y, Shi Y Y, Lan Y B, Guo, S. Vertical distribution and vortex structure of rotor wind field under the influence of rice canopy. Computers and Electronics in Agriculture, 2019; 159: 140-146.

[27] Wang P, Hu L, Zhou Z Y, Yang W S, Liu A M, Luo X W, et al. Wind field measurement for supplementary pollination in hybrid rice breeding using unmanned gasoline engine single-rotor helicopter. Transactions of the CSAE, 2013; 29(3): 54-61. (in Chinese)

[28] Hu L, Zhou Z Y, Luo X W, Wang P, Yan Y A, Li J Y. Development and experiment of a wireless wind speed sensor network measurement system for unmanned helicopter. Transactions of the CSAM, 2014; 45(5): 194-201. (in Chinese)

[29] Wang C L, He X K, Wang X N, Bonds J, Herbst A, Wang Z G, et al. Testing method of spatial pesticide spraying deposition quality balance for unmanned aerial vehicle. Transactions of the CSAE, 2016; 32(11): 54-61 (in Chinese)

[30] Pombeiro R, Mendona R, Rodrigues P, Marques F, Barata J. Water detection from downwash-induced optical flow for a multirotor UAV. 
OCEANS 2015-MTS/IEEE, Washington, USA, 2015; pp.1-6.

[31] Mylapore A R, Schmitz F H. An experimental investigation of ground effect on a quad tilt rotor in hover. Journal of the American Helicopter Society, 2015; 60(1): 1-14.

[32] Guo Q W, Zhu Y Z, Tang Y, Hou C J, He L, Zhuang J J, et al. CFD simulation and experimental verification of the spatial and temporal distributions of the downwash airflow of a quad-rotor agricultural UAV in hover. Computers and Electronics in Agriculture, 2020; 172: 105343. doi: 10.1016/j.compag.2020.105343.

[33] Tang Q, Zhang R R, Chen L P, Xu G, Li L L. High-accuracy, high-resolution downwash flow field measurements of an unmanned helicopter for precision agriculture. Computers and Electronics in Agriculture, 2020; 173: 105390. doi: 10.1016/j.compag.2020.105390.

[34] Lan Y B, Chen S D, Fritz B K. Current status and future trends of precision agricultural aviation technologies. Int J Agric \& Biol Eng, 2017; 10(3): 1-17.

[35] Wu P. Power-train dynamical analysis based on testing and computational modals. Chinese Journal of Construction Machinery, 2013; 11(6): 534-536,541.
[36] Zhang X Y, Lu Z H, Wu S Y, Zhao Y G. An efficient method for time-variant reliability including finite element analysis. Reliability Engineering \& System Safety, 2021; 210: $107534 . \quad$ doi 10.1016/j.ress.2021.107534.

[37] Guo Q, Yao W J, Li W B, Gupta N. Constitutive models for the structural analysis of composite materials for the finite element analysis: a particular review of recent practices. Composite Structures, 2021; 260: 113267. doi: 10.1016/j.compstruct.2020.113267.

[38] Kweon H D, Kim J W, Song O, Oh D. Determination of true stress-strain curve of type 304 and 316 stainless steels using a typical tensile test and finite element analysis. Nuclear Engineering and Technology, 2021; 53: 647-656.

[39] Maeda R, Wang Z L, Ogawa T, Adachi Y. Stress-strain partitioning behavior and mechanical properties of dual-phase steel using finite element analysis. Materials Today Communications, 2020; 25: 101658. doi: 10.1016/j.mtcomm.2020.101658.

[40] Liu H S, Luo D. The research and application of vibration modal test and simulation analysis. Mechanical Management and Development, 2018; 4 : $38-41$.

\section{Appendix}

Statistics of measured down wash wind speeds when CPUAS was at the height of $5.0 \mathrm{~m}$

\begin{tabular}{|c|c|c|c|c|c|c|c|c|c|}
\hline \multirow{2}{*}{ Height/m } & \multirow{2}{*}{$\begin{array}{c}\text { Radial } \\
\text { distance/m }\end{array}$} & \multicolumn{8}{|c|}{ Wind speed $/ \mathrm{m} \cdot \mathrm{s}^{-1}$} \\
\hline & & $O_{1}$ & $\mathrm{O}_{2}$ & $\mathrm{O}_{3}$ & $\mathrm{O}_{4}$ & $O_{5}$ & $O_{6}$ & $O_{7}$ & $O_{8}$ \\
\hline \multirow{8}{*}{0.5} & 0.5 & $4.27 \pm 0.13$ & $3.17 \pm 0.10$ & $4.70 \pm 0.14$ & $7.07 \pm 0.21$ & $7.97 \pm 0.24$ & $7.80 \pm 0.23$ & $4.23 \pm 0.13$ & $7.07 \pm 0.21$ \\
\hline & 1.0 & $4.60 \pm 0.14$ & $5.67 \pm 0.17$ & $3.17 \pm 0.10$ & $5.69 \pm 0.17$ & $7.07 \pm 0.21$ & $7.13 \pm 0.21$ & $5.50 \pm 0.17$ & $6.56 \pm 0.20$ \\
\hline & 1.5 & $6.87 \pm 0.21$ & $4.80 \pm 0.14$ & $2.77 \pm 0.08$ & $3.89 \pm 0.12$ & $6.98 \pm 0.21$ & $5.20 \pm 0.16$ & $2.77 \pm 0.08$ & $5.44 \pm 0.16$ \\
\hline & 2.0 & $4.97 \pm 0.15$ & $3.03 \pm 0.09$ & $0.24 \pm 0.01$ & $2.14 \pm 0.06$ & $6.12 \pm 0.18$ & $3.41 \pm 0.10$ & $3.62 \pm 0.11$ & $3.28 \pm 0.10$ \\
\hline & 2.5 & $2.57 \pm 0.08$ & $1.13 \pm 0.03$ & $0.47 \pm 0.01$ & $2.30 \pm 0.07$ & $3.23 \pm 0.10$ & $2.88 \pm 0.09$ & $2.88 \pm 0.09$ & $2.30 \pm 0.07$ \\
\hline & 3.0 & $0.67 \pm 0.02$ & $1.17 \pm 0.04$ & $0.33 \pm 0.01$ & $1.98 \pm 0.06$ & $1.34 \pm 0.04$ & $1.21 \pm 0.04$ & $1.87 \pm 0.06$ & $2.10 \pm 0.06$ \\
\hline & 3.5 & $0.21 \pm 0.01$ & $0.42 \pm 0.01$ & $0.20 \pm 0.01$ & $2.10 \pm 0.06$ & $1.10 \pm 0.03$ & $0.46 \pm 0.01$ & $0.99 \pm 0.03$ & $0.45 \pm 0.01$ \\
\hline & 4.0 & $0.10 \pm 0.01$ & $0.23 \pm 0.01$ & $0.10 \pm 0.01$ & $0.98 \pm 0.03$ & $0.36 \pm 0.01$ & $0.21 \pm 0.01$ & $0.21 \pm 0.01$ & $0.11 \pm 0.01$ \\
\hline \multirow{7}{*}{1.0} & 0.5 & $5.67 \pm 0.17$ & $4.67 \pm 0.14$ & $4.20 \pm 0.13$ & $4.37 \pm 0.13$ & $7.27 \pm 0.22$ & $6.12 \pm 0.18$ & $5.12 \pm 0.15$ & $4.99 \pm 0.15$ \\
\hline & 1.0 & $3.93 \pm 0.12$ & $2.23 \pm 0.07$ & $6.13 \pm 0.18$ & $5.21 \pm 0.16$ & $7.92 \pm 0.24$ & $4.98 \pm 0.15$ & $5.20 \pm 0.16$ & $5.21 \pm 0.16$ \\
\hline & 1.5 & $3.43 \pm 0.10$ & $1.20 \pm 0.04$ & $1.47 \pm 0.04$ & $2.99 \pm 0.09$ & $6.43 \pm 0.19$ & $4.21 \pm 0.13$ & $4.32 \pm 0.13$ & $5.38 \pm 0.16$ \\
\hline & 2.0 & $1.03 \pm 0.03$ & $1.23 \pm 0.04$ & $0.57 \pm 0.02$ & $1.58 \pm 0.05$ & $5.21 \pm 0.16$ & $2.65 \pm 0.08$ & $2.89 \pm 0.09$ & $3.49 \pm 0.10$ \\
\hline & 3.0 & $1.07 \pm 0.03$ & $0.27 \pm 0.01$ & $0.20 \pm 0.01$ & $0.59 \pm 0.02$ & $1.10 \pm 0.03$ & $1.54 \pm 0.05$ & $1.32 \pm 0.04$ & $0.61 \pm 0.02$ \\
\hline & 3.5 & $0.25 \pm 0.01$ & $0.13 \pm 0.01$ & $0.10 \pm 0.01$ & $0.45 \pm 0.01$ & $0.87 \pm 0.03$ & $0.76 \pm 0.02$ & $0.89 \pm 0.03$ & $0.33 \pm 0.01$ \\
\hline & 4.0 & $0.10 \pm 0.01$ & $0.12 \pm 0.01$ & $0.10 \pm 0.01$ & $0.33 \pm 0.01$ & $0.41 \pm 0.01$ & $0.23 \pm 0.01$ & $0.22 \pm 0.01$ & $0.21 \pm 0.01$ \\
\hline \multirow{8}{*}{1.5} & 0.5 & $6.71 \pm 0.20$ & $5.54 \pm 0.17$ & $3.83 \pm 0.11$ & $3.95 \pm 0.12$ & $7.81 \pm 0.23$ & $6.56 \pm 0.20$ & $5.56 \pm 0.17$ & $4.45 \pm 0.13$ \\
\hline & 1.0 & $6.39 \pm 0.19$ & $4.74 \pm 0.14$ & $5.42 \pm 0.16$ & $4.86 \pm 0.15$ & $7.42 \pm 0.22$ & $6.88 \pm 0.21$ & $5.42 \pm 0.16$ & $4.86 \pm 0.15$ \\
\hline & 1.5 & $6.37 \pm 0.19$ & $3.20 \pm 0.10$ & $3.87 \pm 0.12$ & $2.98 \pm 0.09$ & $7.10 \pm 0.21$ & $6.66 \pm 0.20$ & $4.97 \pm 0.15$ & $5.21 \pm 0.16$ \\
\hline & 2.0 & $5.65 \pm 0.17$ & $2.37 \pm 0.07$ & $1.56 \pm 0.05$ & $1.22 \pm 0.04$ & $6.85 \pm 0.21$ & $5.52 \pm 0.17$ & $3.98 \pm 0.12$ & $4.78 \pm 0.14$ \\
\hline & 2.5 & $3.73 \pm 0.11$ & $1.95 \pm 0.06$ & $2.24 \pm 0.07$ & $0.97 \pm 0.03$ & $4.80 \pm 0.14$ & $4.31 \pm 0.13$ & $2.24 \pm 0.07$ & $3.21 \pm 0.10$ \\
\hline & 3.0 & $3.93 \pm 0.12$ & $1.13 \pm 0.03$ & $1.30 \pm 0.04$ & $0.73 \pm 0.02$ & $4.21 \pm 0.13$ & $3.98 \pm 0.12$ & $1.33 \pm 0.04$ & $2.11 \pm 0.06$ \\
\hline & 3.5 & $1.12 \pm 0.03$ & $0.95 \pm 0.03$ & $0.89 \pm 0.03$ & $0.23 \pm 0.01$ & $1.22 \pm 0.04$ & $1.99 \pm 0.06$ & $0.89 \pm 0.03$ & $0.34 \pm 0.01$ \\
\hline & 4.0 & $0.53 \pm 0.02$ & $0.40 \pm 0.01$ & $0.55 \pm 0.02$ & $0.17 \pm 0.01$ & $0.63 \pm 0.02$ & $0.67 \pm 0.02$ & $0.44 \pm 0.01$ & $0.17 \pm 0.01$ \\
\hline \multirow{7}{*}{2.0} & 0.5 & $7.75 \pm 0.23$ & $6.40 \pm 0.19$ & $3.45 \pm 0.10$ & $3.80 \pm 0.11$ & $8.85 \pm 0.27$ & $8.21 \pm 0.25$ & $6.21 \pm 0.19$ & $4.89 \pm 0.15$ \\
\hline & 1.0 & $8.84 \pm 0.27$ & $7.25 \pm 0.22$ & $4.70 \pm 0.14$ & $4.50 \pm 0.14$ & $9.94 \pm 0.30$ & $8.88 \pm 0.27$ & $5.88 \pm 0.18$ & $4.56 \pm 0.14$ \\
\hline & 1.5 & $9.30 \pm 0.28$ & $5.20 \pm 0.16$ & $6.26 \pm 0.19$ & $2.96 \pm 0.09$ & $10.4 \pm 0.31$ & $9.30 \pm 0.28$ & $5.45 \pm 0.16$ & $4.88 \pm 0.15$ \\
\hline & 2.5 & $6.12 \pm 0.18$ & $2.89 \pm 0.09$ & $3.80 \pm 0.11$ & $0.63 \pm 0.02$ & $7.22 \pm 0.22$ & $7.87 \pm 0.24$ & $3.80 \pm 0.11$ & $3.98 \pm 0.12$ \\
\hline & 3.0 & $6.78 \pm 0.20$ & $2.00 \pm 0.06$ & $2.40 \pm 0.07$ & $0.87 \pm 0.03$ & $7.88 \pm 0.24$ & $7.12 \pm 0.21$ & $2.40 \pm 0.07$ & $2.79 \pm 0.08$ \\
\hline & 3.5 & $4.50 \pm 0.14$ & $1.76 \pm 0.05$ & $1.67 \pm 0.05$ & $0.60 \pm 0.02$ & $3.10 \pm 0.09$ & $3.34 \pm 0.10$ & $1.22 \pm 0.04$ & $1.09 \pm 0.03$ \\
\hline & 4.0 & $1.10 \pm 0.03$ & $0.67 \pm 0.02$ & $1.00 \pm 0.03$ & $0.20 \pm 0.01$ & $0.91 \pm 0.03$ & $2.20 \pm 0.07$ & $0.38 \pm 0.01$ & $0.31 \pm 0.01$ \\
\hline \multirow{8}{*}{2.5} & 0.5 & $6.98 \pm 0.21$ & $6.69 \pm 0.20$ & $3.64 \pm 0.11$ & $3.88 \pm 0.12$ & $8.08 \pm 0.24$ & $7.88 \pm 0.24$ & $6.87 \pm 0.21$ & $6.41 \pm 0.19$ \\
\hline & 1.0 & $9.24 \pm 0.28$ & $8.25 \pm 0.25$ & $5.06 \pm 0.15$ & $4.68 \pm 0.14$ & $10.12 \pm 0.30$ & $9.45 \pm 0.28$ & $6.43 \pm 0.19$ & $5.89 \pm 0.18$ \\
\hline & 1.5 & $9.31 \pm 0.28$ & $7.26 \pm 0.22$ & $5.06 \pm 0.15$ & $2.97 \pm 0.09$ & $9.24 \pm 0.28$ & $9.44 \pm 0.28$ & $5.06 \pm 0.15$ & $5.88 \pm 0.18$ \\
\hline & 2.0 & $8.99 \pm 0.27$ & $6.25 \pm 0.19$ & $3.47 \pm 0.10$ & $1.04 \pm 0.03$ & $9.10 \pm 0.27$ & $9.12 \pm 0.27$ & $4.47 \pm 0.13$ & $4.96 \pm 0.15$ \\
\hline & 2.5 & $5.98 \pm 0.18$ & $4.44 \pm 0.13$ & $3.02 \pm 0.09$ & $0.80 \pm 0.02$ & $6.25 \pm 0.19$ & $6.21 \pm 0.19$ & $3.29 \pm 0.10$ & $3.77 \pm 0.11$ \\
\hline & 3.0 & $4.32 \pm 0.13$ & $3.16 \pm 0.09$ & $1.85 \pm 0.06$ & $0.80 \pm 0.02$ & $4.33 \pm 0.13$ & $3.89 \pm 0.12$ & $2.00 \pm 0.06$ & $2.10 \pm 0.06$ \\
\hline & 3.5 & $2.01 \pm 0.06$ & $1.89 \pm 0.06$ & $1.28 \pm 0.04$ & $0.42 \pm 0.01$ & $1.93 \pm 0.06$ & $1.98 \pm 0.06$ & $1.11 \pm 0.03$ & $1.08 \pm 0.03$ \\
\hline & 4.0 & $1.06 \pm 0.03$ & $0.87 \pm 0.03$ & $0.78 \pm 0.02$ & $0.19 \pm 0.01$ & $1.11 \pm 0.03$ & $1.23 \pm 0.04$ & $0.78 \pm 0.02$ & $0.45 \pm 0.01$ \\
\hline
\end{tabular}

Note: The values are represented as mean \pm standard deviation. 